\title{
HEMATOPOIETIC STEM CELL TRANSPLANTATION FOR PEDIATRIC CHRONIC MYELOID LEUKEMIA
}

\author{
Roseane Vasconcelos Gouveia ${ }^{1}$, Luciana dos Santos Domingues ${ }^{1}$, Paola Azenha Milani Soriano ${ }^{1}$, \\ Antonio Vaz de Macedo ${ }^{2,3}$ \\ 1. Pediatric Bone Marrow Transplant Unit, Hospital Samaritano, São Paulo, SP, Brazil. \\ 2. Hematology Clinic, Hospital da Polícia Militar, Belo Horizonte, MG, Brazil \\ 3. Bone Marrow Transplant Unit, Hospital Luxemburgo, Instituto Mário Penna, Belo Horizonte, MG, Brazil.
}

Correspondence to: antoniovmac@hotmail.com

\begin{abstract}
Chronic myeloid leukemia (CML) accounts for approximately 2 to $3 \%$ of all pediatric leukemias. Compared to adults, children tend to present with more aggressive features, such as higher leukocyte counts and massive splenomegaly, and are more likely to be diagnosed with advanced stage disease. Before the advent of tyrosine kinase inhibitors, a couple of decades ago, allogeneic hematopoietic stem cell transplantation (allo-HSCT) was the mainstay of treatment for this disease. This, however, was associated with considerable treatment-related morbidity and mortality. Even so, despite its secondary and somewhat limited indication today, allo-HSCT remains an important alternative and the only curative treatment for CML. In 2020, the Brazilian Group for Pediatric Bone Marrow Transplantation of the Brazilian Society for Blood and Marrow Transplantation and Cellular Therapy (SBTMO) convened a task force to provide evidence-based guidance on the use of allo-HSCT for the appropriate management of childhood CML, the results of which are presented here.
\end{abstract}

Keywords: Hematopoietic Stem Cell Transplantation, Tyrosine Kinase Inhibitors, Chronic Myeloid Leukemia, Childhood, Pediatric, Consensus Guidelines.

Chronic myeloid leukemia (CML) accounts for approximately 2 to $3 \%$ of all childhood and adolescent (under 15 years old) leukemias ${ }^{1}$. These patients tend to present with more aggressive features, such as higher leukocyte counts and massive splenomegaly, and are more likely to be diagnosed with advanced stage disease ${ }^{1}$. Pediatric CML presents the same morphologic, cytogenetic and molecular features observed in adult CML. As such, it is characterized by the presence of the Philadelphia chromosome $(\mathrm{Ph}+)$, which results from a reciprocal translocation between the long arms of chromosomes 9 and 22 [t $(9 ; 22)(q 34 ; q 11)]$, which leads to the BCR-ABL fusion gene. This rearrangement encodes a new protein, with uncontrolled tyrosine kinase activity ${ }^{2}$.

Despite their acknowledged applicability in the adult population, adult risk scores for CML cannot be applied to children, with the exception of the one defined by the European Treatment and Outcome Study, which is able to predict progression and longterm event free survival (EFS), but not overall survival (OS) $)^{1,3,4}$. Therefore, risk scores are not commonly used to guide treatment in pediatric patients with this disease.

Ever since the introduction of tyrosine kinase inhibitors (TKIs) in pediatric $\mathrm{Ph}+\mathrm{CML}$, as in adults, notable changes have been observed in EFS and progression, as well as in the indication for hematopoietic stem cell transplantation (HSCT)4-6. Before the advent of TKIs, a couple of decades ago, allogeneic HSCT was the mainstay of treatment for this disease; even so, despite its secondary and somewhat limited indication today, it remains an important alternative and the only curative treatment for pediatric CML. 
One of the major caveats of TKI-based therapy is that it should be used continuously, maybe lifelong, although this is still an unsolved issue. Moreover, TKls are associated with a number of side effects, some of which are well known, such as growth delay and endocrine disorders, among others, not to mention potential long-term events ${ }^{7,8}$. Besides, the most appropriate approach to assessing treatment response remains unclear, and prospective studies are needed to better define the optimal timing for treatment discontinuation $1,5,6,9,10$. Allogeneic HSCT may thus help circumvent the long-term effects of indefinite TKI therapy in this population. One should, however, ponder the trade-off between its curative potential and its myriad acute and late toxicities when considering this treatment strategy.

Overall, outcomes of allogeneic HSCT tend to be superior in childhood CML as compared to those of adults with this disease, with an OS rate between 45 and $87 \% 11-14$. Some of the most favorable results may be explained by the improvement in supportive care measures and the use of reduced intensity conditioning regimens (RIC), adopted with a view to reducing the mortality risk associated with these procedures.

The choice of graft source might alter the results of HSCT for CML. A retrospective analysis of the Center for International Blood and Marrow Transplant (CIBMTR) showed worst EFS rates when peripheral blood stem cells (PBSC) were used when compared to bone marrow (BM) in children. Although the incidence of acute graft-versus-host disease (GVHD) was similar between children and adults, chronic GVHD rates were also higher in the group that used PBSC as stem cell source ${ }^{15}$.

\section{INDICATIONS FOR HSCT IN CML}

In a study published in Leukemia in 2016, including 669 patients (among whom only 14 were younger than 20 years of age), 427 were eligible for transplant and randomized between drug therapy and HSCT, depending on related donor availability. The OS of the patients who underwent HSCT was $76 \%$ against $69 \%$ in the drug therapy arm. Additionally, superior rates of molecular remission were noted in the HSCT group (56\% vs. $39 \%$ ), and $56 \%$ of the HSCT patients were no longer in need of drug treatment, as compared to only $6 \%$ of those in the non-transplant group ${ }^{16}$.

There are no robust studies to date in the pediatric population comparing TKIs and HSCT in the treatment of CML. As a rule, treatment is similar to the one applied in adults, where HSCT is indicated after failure of a second generation TKI or in advanced stage (accelerated and blast phase) disease17. In specific cases, HSCT may be indicated after failure of a first line TKI (imatinib mesylate), or when there is a T315I mutation ${ }^{17}$. As for third line TKIs (ponatinib), further studies are needed to better define their efficacy and safety in this population. As previously mentioned, the possibility of adverse events and of poor adherence to the long-term use of TKI, coupled with the potential for curing the disease with HSCT, should be carefully weighed and conditioned upon shared decision-making with the patient and his/her family, on a case-by-case basis, when choosing the best treatment approach for this population 18-22.

In summary, the main indications for HSCT in children with CML are17:

1) Accelerated phase (AP) or blast phase (BP) at the time of diagnosis;

2) Progression to AP or BP. T315I mutation is associated with poor prognosis; children with this mutation may rapidly progress to $\mathrm{BP}$; treatment failure with 1st (imatinib) and 2nd generation (dasatinib, nilotinib) TKI; benefits of the use of 3rd generation TKI are not well known in this population;

3) Poor adherence to TKI treatment (upon discussion of the possible benefit of HSCT in this situation);

4) Severe toxicities related to the use of TKIs.

\section{CONDITIONING REGIMEN}

A recent prospective, non-randomized study from a Japanese group compared results between RIC HSCT plus imatinib vs. imatinib alone in the treatment of young adults (including children) with $\mathrm{CML}$ in early ( $<12$ months) chronic phase (CP) or late $(\geq 12$ months) CP, with a median age of 34 (11-49) years23. In this study, patients undergoing HSCT were conditioned with fludarabine $30 \mathrm{mg} / \mathrm{m} 2 /$ day from D-10 to $\mathrm{D}-5$, oral busulfan $4 \mathrm{mg} / \mathrm{kg} /$ day or intravenous busulfan $3.2 \mathrm{mg} / \mathrm{kg} /$ day from D-6 to D-5, and Thymoglobulin $^{\oplus}$ - rabbit anti-thymocyte globulin (Fresenius ${ }^{\oplus}$ ) $5 \mathrm{mg} / \mathrm{kg} /$ day from D-4 to D-1. GVHD prophylaxis consisted of cyclosporine, mycophenolate mofetil (MMF), and methotrexate. In this group, imatinib was also used at a dose of $400 \mathrm{mg} /$ day, three to 12 months before HSCT, and, as a prophylactic drug, at a dose of $300 \mathrm{mg}$ to $400 \mathrm{mg} /$ day, from D+100 until 1 year after transplant. Prolonged treatment with a higher dose of imatinib was used for patients with 
persistent residual disease or hematologic or cytogenetic relapse. In these cases, the drug was only discontinued 12 months after complete cytogenetic remission. Patients in the imatinib-only group took the usual 400mg/day dosage, with adjustments according to toxicity and response. The estimated 10-year OS and EFS were comparable between the groups. In the late CP CML group, although both treatments resulted in similar survival, a worse 10-year EFS was noted in the imatinib-alone group as compared to the HSCT + TKI group (40.8 vs. $66.7 \%, p=0.047$, respectively). Of note, HSCT patients with higher European Group for Blood and Marrow Transplantation (EBMT) risk scores had a worse OS than those with lower scores (69.2 vs. $92.9 \%, p=0.04$ ). The authors concluded that HSCT in combination with imatinib seems more cost-effective than imatinib alone and should be considered as an appropriate option, particularly for patients with low EBMT risk scores and for whom cure of $C M L$ is the ultimate goal.

Regarding haploidentical HSCT for pediatric CML, there are only a few studies available to date, all of which are limited to retrospective analyses of a small number of cases. Hence, further studies are needed to better define the role of this transplant modality in this population ${ }^{24,25}$.

\section{RECOMMENDATIONS:}

1. Related donor HSCT: fludarabine + busulfan (RIC) 23 or busulfan + cyclophosphamide (myeloablative)12. GVHD prophylaxis: cyclosporine + methotrexate.

2. Unrelated donor HSCT: fludarabine + busulfan + anti-thymocyte globulin (RIC) 23 or busulfan + cyclophosphamide + anti-thymocyte globulin (myeloablative)12. GVHD prophylaxis: cyclosporine + methotrexate.

\section{USE OF TYROSINE KINASE INHIBITORS AFTER HSCT}

In the study by Zhao $Y$ et al., 2017, imatinib was used prophylactically at a dose of $300 \mathrm{mg}$ to $400 \mathrm{mg} /$ day from D+100 until 1 year after HSCT. In patients with persistent residual disease, or with hematologic or cytogenetic relapse, a higher dose of imatinib $(600 \mathrm{mg} /$ day) was used for at least 1 year after achieving complete cytogenetic remission ${ }^{23}$.

In case of disease progression while using imatinib prior to transplant, one should switch to another generation TKI (dasatinib, nilotinib, or other), according to one's clinical history and mutational status.

\section{STRATEGIES TO AVOID DISEASE RELAPSE}

A few strategies can be used to avoid disease relapse after HSCT, as presented in the ASH Educational Program published in Hematology in 2018 ${ }^{17}$ :

1. Minimize pre-transplant disease burden;

2. Optimize conditioning regimen;

3. Optimize the graft-versus-leukemia (GVL) effect: minimize post-transplant immunosuppression and use prophylactic donor lymphocyte infusion (DLI).

Importantly, disease status should be regularly monitored in children with CML, with molecular and cytogenetic studies, following the National Comprehensive Cancer Network (NCCN), European Leukemia Net (ELN), or European Society of Medical Oncology (ESMO) guidelines, both pre- and post-transplant, since this will allow for appropriate and timely interventions according to optimal treatment response assessments ${ }^{5}$.

\begin{tabular}{c|c|c}
\hline Chronic myeloid leukemia & Allogeneic HSCT & Autologous HSCT \\
\hline Chronic phase & Yes (standard of care, clinical evidence) & No \\
\hline 1st chronic phase refractory to TKIs & Yes (standard of care, clinical evidence) & No \\
\hline 1st chronic phase intolerant to TKIs & Yes (standard of care, clinical evidence) & No \\
\hline Accelerated phase & Yes (standard of care, clinical evidence) & No \\
\hline Blast phase & Yes (standard of care, clinical evidence) & No
\end{tabular}




\section{REFERENCES}

1. Hijiya N, Suttorp M. How I treat chronic myeloid leukemia in children and adolescents. Blood. 2019;133(22):2374-2384.

2. Suttorp M, Millot F. Treatment of Pediatric Chronic Myeloid Leukemia in the Year 2010: Use of Tyrosine Kinase Inhibitors and Stem-Cell Transplantation. Hematology Am Soc Hematol Educ Program. 2010;2010:368-6.

3. Millot F, Guilhot J, Suttorp M, Güneş AM, Sedlacek $P$, De Bont $E$, et al. Prognostic discrimination based on the EUTOS long-term survival score within the International Registry for Chronic Myeloid Leukemia in children and adolescents. Haematologica 2017;102(10):1704-1708.

4. NCCN Guidelines Version 3.2020 Chronic Myeloid Leukemia. National Comprehensive Cancer Network. Available at: https://www.nccn. org/patients/guidelines/content/PDF/cml-patient.pdf Accessed: 20th September, 2020.

5. Shanmuganathan N, Hughes TP. Molecular monitoring in CML: How deep? How often? How should it influence therapy? Blood. 2018;132(20):2125-2133.

6. Björkholm M, Ohm L, Eloranta S, Derolf A, Hultcrantz M, Sjoberg J, et al. Success Story of Targeted Therapy in Chronic Myeloid Leukemia: A Population-Based Study of Patients Diagnosed in Sweden from 1973 to 2008. J Clin Oncol. 2011;29(18): 2514-2520.

7. Bansal D, Shava U, Varma N, Trehan A, Marwaha RK. Imatinib Has Adverse Effect on Growth in Children with Chronic Myeloid Leukemia. Pediatr Blood Cancer. 2012;59(3):481-484

8. Samis J, Lee P, Zimmerman D, Arceci RJ, Suttorp $M$, Hijiya N. Recognizing endocrinopathies associated with tyrosine kinase inhibitor therapy in children with chronic myelogenous leukemia. Pediatr Blood Cancer. 2016;63(8):1332-1338.

9. De la Fuente J, Baruchel A, Biondi A, Bont E, Dresse MF, Suttorp M, et al - International BFM Group (iBFM) Study Group Chronic Myeloid Leukaemia Committee. Managing children with chronic myeloid leukaemia (CML) Recommendations for the management of CML in children and young people up to the age of 18 years. British Journal of Haematology. 2014;167(1): 33-47.
10. Giona F, Santopietro M, Menna G, Putti MC, Micalizzi C, Santoro N, et al. Real-Life Management of Children and Adolescents with Chronic Myeloid Leukemia: The Italian Experience. Acta Haematol. 2018;140(2):105-111.

11. Chaudhury S, Sparapani R, Hu Z, Nishihori T, Abdel-Azim $\mathrm{H}$, Malone A, et al. Outcomes of allogeneic hematopoietic stem cell transplantation in children and young adults with chronic myeloid leukemia: a CIBMTR cohort analysis. Biol Blood Marrow Transplant. 2016;22(6):1056-1064.

12. Suttorp M, Claviez A, Bader P, Peters C, Gadner $\mathrm{H}$, Ebell $\mathrm{W}$, et al. Allogeneic stem cell transplantation for pediatric and adolescent patients with CML: results from the prospective trial CMLpaed I. Klin Padiatr. 2009;221(6):351-357.

13. Cwynarski $K$, Roberts IAG, lacobelli $S$, van Biezen A, Brand R, Devergie A, et al; Paediatric and Chronic Leukaemia Working Parties of the European Group for Blood and Marrow Transplantation. Stem cell transplantation for chronic myeloid leukemia in children. Blood. 2003;102(4):1224-1231.

14. Muramatsu H, Kojima S, Yoshimi A, Atsuta $Y$, Kato K, Nagatoshi Y, et al. Outcome of 125 children with chronic myelogenous leukemia who received transplants from unrelated donors: the Japan Marrow Donor Program. Biol Blood Marrow Transplant. 2010;16(2):231-238.

15. Eapen M, Horowitz M M, Klein J P, Champlin R E, Loberiza Jr F R, Ringdén O, et al. Higher mortality after allogeneic peripheral blood transplantation compared with bone marrow in children and adolescents: the histocompatibility and alternate stem cell source working committee of the international bone marrow transplant registry. J Clin Oncol. 2004;22(24):4872-4880.

16. Gratwohl A, Pfirrmann M, Zander A, Kröger N, Beelen D , Novotny J, et al. German CML Study Group. Long-term outcome of patients with newly diagnosed chronic myeloid leukemia: a randomized comparison of stem cell transplantation with drug treatment. Leukemia. 2016;30(3): 562-569.

17. Craddock, CF. We do still transplant CML, don't we? Hematology Am Soc Hematol Educ Program. 2018;2018(1):177-184. 
18. Suttorp M, Schulze P, Glauche I, Gohdring G, von Neuhoff N, Metzler M, et al. Frontline imatinib treatment in children and adolescents with chronic myeloid leukemia: results from a phase III trial. Leukemia. 2018;32(7):1657-1669.

19. Millot F, Baruchel A, Guilhot J, Petit A, Leblanc $T$, Bertrand $Y$, et al. Imatinib is effective in children with previously untreated chronic myelogenous leukemia in early chronic phase: results of the French national phase IV trial. J Clin Oncol. 2011;29(20): 2827-2832.

20. Gore L, Kearns PR, de Martino ML, Souza CA, Bertrand $\mathrm{Y}$, Hijiya $\mathrm{N}$, et al. Dasatinib in pediatric patients with chronic myeloid leukemia in chronic phase: results from a phase II trial. J Clin Oncol. 2018; 36(13):1330-1338.

21. Hijiya N, Maschan A, Rizzari C, Shimada H, Dufour $\mathrm{C}$, Goto $\mathrm{H}$, et al. Phase 2 study of nilotinib in pediatric patients with Philadelphia chromosome-positive chronic myeloid leukemia. Blood. 2019;134(23):2036-2045.

22. Mancini J, Simeoni MC, Parola N, Clement A, Vey $\mathrm{N}$, Sirvent N, et al. Adherence to leukemia maintenance therapy:a comparative study among children, adolescents, and adults. Pediatr Hematol Oncol. 2012;29(5):428-439.

23. Zhao Y, Wang J, Luo Y, Shi J, Zheng W, Tan Y, et al. Reduced-intensity allogeneic hematopoietic stem cell transplantation combined with imatinib has comparable event-free survival and overall survival to long-term imatinib treatment in young patients with chronic myeloid leukemia. Ann Hematol. 2017;96(8): 1353-1360.

24. Berger M, Lanino E, Cesaro S, Zecca M, Vassallo $E$, Faraci $M$, et al. Feasibility and Outcome of Haploidentical Hematopoietic Stem Cell Transplantation with Post-Transplant High-Dose Cyclophosphamide for Children and Adolescents with Hematologic Malignancies: An AIEOP-GITMO Retrospective Multicenter Study. Biol Blood Marrow Transplant. 2016;22(5):902-909.

25.González-Llano O, González-López EE, Ramírez-Cázares AC, Marcos-Ramírez ER, Ruiz-Argüelles GJ, Gómez-Almaguer D. Haploidentical peripheral blood stem cell transplantation with posttransplant cyclophosphamide in children and adolescents with hematological malignancies. Pediatr Blood Cancer. 2016;3(11):2033-2037. 